P499 SINGLE-FIBRE EMG ANALYSIS OF THE MATURATION OF THE NEUROMUSCULAR ENDPLATE DURING REINNERVATION AFTER TRAUMATIC NERVE INJURY

Philip Broser*. Kinderspital, Sankt Gallen, Switzerland

10.1136/archdischild-2019-epa.835

Nerve injuries are a common complication of upper extremities fractures. Especially the long arm nerves are prone to injuries. After such an injury the muscles are denervated. Fortunately, if nerves are injured but the continuity of the nerve shed is intact a regeneration by spreading of the axonal fibres starts early after the injury and finally a new neuromuscular connection - a neuromuscular endplate- is reestablished. Till today it is unknown whether the newly formed neuro-muscular endplate is functional equivalent to the original. During the last years a new technic became routinely available in paediatric neurology to study the neuro-muscular endplate: stimulated single-fibre-EMG. This technic allows a precise and objective assessment of the neuro-muscular connection. At our hospital we care together with our colleagues from the paediatric surgery department for children with fractures and nerve injuries. During the last years we have adopted the technique of single fibre EMG and included it into our clinical repertoire for traumatic nerve injuries.

It has been previously hypothesis that the newly formed neuromuscular endplate is not as reliable as the original one. This fact would have major clinical implications for the support and training after nerval injuries. To address this question, we have set up this pilot project to conduct routine measurements of the recovering nerve using single fibre-EMG. Our preliminary data shows, that these measurements can be reliable conducted in the pediatric population and we are now in the process of applying for ethical approval to analyse a larger cohort.

\section{P500 SANDIFER SYNDROME: PRESENTATION, INVESTIGATION AND MANAGEMENT}

Irina Mindlina*. University of Cambridge, Cambridge, UK

10.1136/archdischild-2019-epa.836

Background Sandifer syndrome is a rare complication of gastro-oesophageal reflux disease (GORD) when a patient presents with extraoesophageal symptoms, typically neurological. Although its pathophysiology is not completely understood, one explanation is that neurological manifestations are the consequence of vagal reflex with the reflex centre in nucleus tractus solitarii. The goal of this study was to review the existing literature and describe a typical presentation and most appropriate investigations and management for the Sandifer syndrome.

Methods A comprehensive literature search was performed via PubMed, Cochrane Library and NHS Evidence databases. 27 cases and observational studies were identified.

Results The literature demonstrates that presenting symptoms of Sandifer's may include any combination of abnormal movements and/or positioning of head, neck, trunk and upper limbs, seizure-like episodes, ocular symptoms, irritability, developmental and growth delay, iron-deficiency anaemia.
In terms of diagnostic procedures, 24-hour oesophageal $\mathrm{pH}$ monitoring was positive in all the cases of Sandifer's where it was performed, while upper GI endoscopy \pm biopsy and barium swallow were diagnostic only in a subset of cases.

A range of treatment options were applied in the reviewed literature, including dietary changes, pharmacological management, enteral tube feeding, and surgical approach. These treatment options are consistent with the 2015 NICE guidelines on management of GORD in children and young people.

Successful treatment of the underlying gastro-oesophageal pathology led to a complete or near-complete resolution of the neurological symptoms in all of the cases.

Discussion As Sandifer syndrome is driven by the underlying gastro-oesophageal reflux, it is not surprising that its investigations and management showed to be consistent with those of GORD. It is evident from the literature that many patients were originally misdiagnosed with various neuropsychiatric diagnoses that led to unnecessary testing and ineffective medications with significant side effects. Earlier diagnosis of Sandifer's would have allowed to avoid them.

\section{P501 FUNCTIONAL HEMISPHEROTOMY AS A TREATMENT OF CHOICE IN REFRACTORY RASMUSSEN SYNDROME}

Bojan Marjanovic*, Vladimir Pavlovic. Primary Health Center, Lazarevac, Serbia

10.1136/archdischild-2019-epa.837

Rasmussen syndrome is a subacute inflammatory encephalitis and one of the causes of continual partial seizures. The onset of convulsive attacks is usually preceded by an episode of non-specific fever, usually of viral origin (eg, CMV infection). Attacks are usually partial, long-lasting and frequent. It most often occurs in children under 10 years of age. EEG findings show diffuse paroxysmal activity. The disease is progressive, with development of various neurological deficits (hemiplegia, hemianopsia, and aphasia). Here we present a case of a 3 year-old girl, with normal early psychomotor development, who had the first absence-type convulsive attack during her holidays in Greece in 2016. Treatment with valproic acid was initiated. In the next two months, two more seizures followed, accompanied with fever (eyes fixation, arm and leg swings, squinting, non-response to calls for 10 seconds). Both times she was hospitalized, and during the second hospitalization, EEG showed the existence of generalized epileptiform changes. MR of the endocranium was normal. Due to poor control of attacks and their frequency, new antiepileptics (lamiktal, carbamazepine, topamax) have been introduced into the therapy. In March 2017, new MR of the endocranium showed the excistence of a hypotrophy of the left hemisphere and descreet left-sided Walerian degeneration, when diagnosis of Rasmussen's syndrome was suspected. Further diagnostic procedures included immunological examination of the liquor and the presence of oligoclonal IgG strains in the liquor (LGI1, AMPA, GABA-B) was confirmed. Anti-NMDA autoantibodies were negative, and therapy with specific immunoglobulins was administered. Corticosteroids were also administered during the exacerbations. In a further course of the disease, a clinical finding reveals a worsening of neurological status (spastic right-sided hemiparesis, facial nerve paralysis and dysarthria). At the end of 2017 , the girl had an average of $4-5$ convulsive 